\title{
Author Correction: Selection of a subspecies-specific diterpene gene cluster implicated in rice disease resistance
}

Chuansong Zhan (1), Long Lei, Zixin Liu, Shen Zhou, Chenkun Yang, Xitong Zhu, Hao Guo, Feng Zhang, Meng Peng (1),

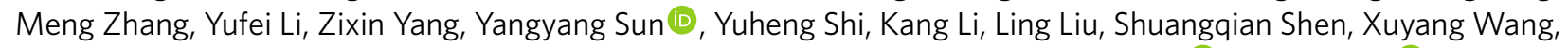
Jiawen Shao, Xinyu Jing, Zixuan Wang, Yi Li, Tomasz Czechowski, Morifumi Hasegawa®D, lan Graham (D), Takayuki Tohge, Lianghuan Qu, Xianqing Liu, Alisdair R. Fernie (iD, Ling-Ling Chen (1D), Meng Yuan (1) and Jie Luo (D)

Correction to: Nature Plants https://doi.org/10.1038/s41477-020-00816-7, published online 7 December 2020.

In the version of this Letter originally published, the surname of the author Morifumi Hasegawa was misspelt as 'Hasegewa'. Also, the author Jie Luo should not have been affiliated with the National Key Laboratory of Crop Genetic Improvement and National Center of Plant Gene Research (Wuhan); they should only have been affiliated with the College of Tropical Crops at Hainan University. These errors have been corrected.

Published online: 16 December 2020

https://doi.org/10.1038/s41477-020-00838-1

(c) The Author(s), under exclusive licence to Springer Nature Limited 2020 